\title{
A Pattern Recognition Algorithm for Continuous Tone Images
}

\author{
Kshitiz Agarwal \\ Jaypee Inst. of Info. Tech. \\ 804, Sai Kirpa Appartment, sector-11, \\ Vasundhara, Ghaziabad, India
}

\author{
Karm Veer Arya \\ AVB-Indian Inst. of Info. Tech.\& \\ Management \\ Morena Link road, Gwalior, India
}

\begin{abstract}
This paper describes a unique method of recognizing common patterns among various images. It makes use of signatures of images which enables it to overcome the problem of different features ordering and appearance of noisy and less expressive features. It discusses two different methods of finding common set of data among various signatures. It has been then concluded that the proposed algorithm would be very suitable for continuous tone images with high contrast patterns.
\end{abstract}

\section{INTRODUCTION}

Pattern recognition is the process of finding common features among various patterns or images. There has been a considerable growth in this area [1]. Its applications are in remote sensing, face recognition, etc. The motive of research in this area is to find maximum common features among images along with elimination of problems of noisy effects and different order of features.

One of the main issues to be considered is time consumption. Principle component analysis approach [2] has been improved several times in order to decrease the time of execution, keeping the efficiency higher. This approach balances time and efficiency of the given problem. It forms signatures of various images from which common set of data is extracted by using depth first search (DFS) and breadth first search (BFS) techniques [12]. It is the content in each signature which decides time required and the efficiency for this approach. Higher the content level, better would be the efficiency and more time would be consumed and vice-versa.

Rest of the paper is organized as follows. Section II shows various works related to the prescribed problem. Section III explains the structure of signature shows various properties carried by common data set. DFS and BFS techniques are discussed in section IV which is then followed by conclusion in the last section.

\section{RELATED WORK}

Various techniques have been developed for this. Principle component analysis (PCA) involves linear combination of set of features among various patterns [2]. This technique has been further improved by S. Chen [3], K. Than [4], X. Tan [5] and K.V. Kumar [6]. PCA can also be applied to 3 independent source images [7] which can be derived through blind source separation procedure like ICA [8], [9]. To solve the problem related to objects belonging to the concept of "cold and warm", "good and bad", etc. fuzzy theory was adopted by Zhang Xiayu [10]. Hong Lian proposed a naval adaptive feature learning algorithms [11] to eliminate noisy effects.

Our proposed method makes use of documented form of an image which describes an approximate idea of colors and positions of its pixels. This form, regarded as its signature, is used to find every common feature and helps to sort out various obstructions.

\section{3. PROPERTIES OF COMMON FEATURES}

Any pixel in an image can be recognized with its color value and position. Ranges of all colors for every bit depth image have been formed such that each range carry almost similar colors and no two ranges have a common color. Now, it is a common property of continuous tone images to have correlated neighboring pixels. So, every row of an image can be represented in the form of series of ranges, each represented by a unique character and accompanied with initial and final position of pixels occupying the colors belonging to that particular range. This new documented representation is its signature carrying same number of rows as that of image.

A feature in an image can be defined as a small or large group of pixels having a particular dimension. Dimension include value of each of its pixel, number of continuous correlated pixels, number of rows along with their positions, position of one row with respect to another (relative position) etc. For common features, everything would be same except their pixels positions which can vary for different feature ordering.

Consider two images $\mathrm{A}$ and $\mathrm{B}$ with $\mathrm{N}$ and $\mathrm{M}$ number of rows $\left(\mathrm{A}_{4}\right)$ respectively. Suppose every $i_{\text {th }}$ row in $A$ has $a_{i}$ pixels and in $B$ has $b_{i}$ pixels. Let $r_{i 1}, r_{i 2} \ldots \ldots r_{\text {ini }}$ be its ranges in every $i_{\text {th }}$ row. For every $r_{k}$ range, $u_{k}$ and $v_{k}$ tell its starting and ending position respectively. Signature of A is

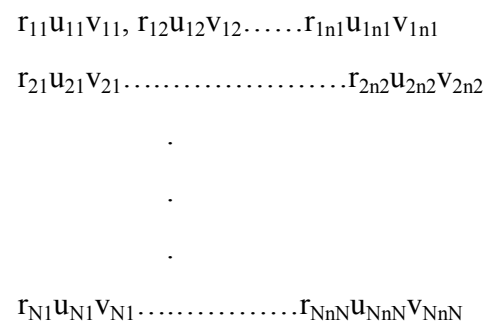


For $i_{\text {th }}$ row, number of pixels $\left(A_{1}\right)$ is $\sum\left(\left(v_{i j}-u_{i j}\right)+1,1<=j<=n i\right)$, number of ranges $\left(A_{2}\right)$ is ni and number of pixels $\left(A_{3}\right)$ in $j_{\text {th }}$ range is $\left(\left(\mathrm{v}_{\mathrm{ij}}-\mathrm{u}_{\mathrm{ij}}\right)+1\right)$.

Similarly, signature of B can be

$$
\begin{aligned}
& \mathrm{R}_{11} \mathrm{u}_{11} \mathrm{v}_{11}, \mathrm{R}_{12} \mathrm{u}_{12} \mathrm{v}_{12} \ldots \ldots \mathrm{R}_{1 \mathrm{~m} 1} \mathrm{u}_{1 \mathrm{~m} 1} \mathrm{v}_{1 \mathrm{~m} 1} \\
& \mathrm{R}_{21} \mathrm{u}_{21} \mathrm{v}_{21} \ldots \ldots \ldots \ldots \ldots \mathrm{R}_{2 \mathrm{~m} 2} \mathrm{u}_{2 \mathrm{~m} 2} \mathrm{v}_{2 \mathrm{~m} 2}
\end{aligned}
$$

$\mathrm{R}_{\mathrm{M} 1} \mathrm{u}_{\mathrm{M} 1} \mathrm{v}_{\mathrm{M} 1} \ldots \ldots \ldots . \mathrm{R}_{\mathrm{MmM}} \mathrm{u}_{\mathrm{MmM}} \mathrm{v}_{\mathrm{MmM}}$

For ith row, $A_{1}$ is $\sum\left(\left(v_{i j}-u_{i j}\right)+1,1<=j<=m i\right), A_{2}$ is mi and $A_{3}$ in $\mathrm{j}_{\mathrm{th}}$ range is $\left.\left(\mathrm{v}_{\mathrm{ij}}-\mathrm{u}_{\mathrm{ij}}\right)+1\right)$.

So, signature is like a matrix $a_{i j}$ where ' $i$ ' is $A_{4}$ and ' $j$ ' is max $\left({ }^{i} A_{2}\right.$, $1<=\mathrm{i}<=\mathrm{A}_{4}$ ).

Now assume that A and B hold a common feature. Suppose that in $\mathrm{A}$, its signature is given by

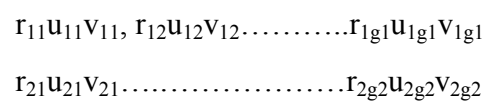

Where, $\mathrm{G}<\mathrm{N}$ and gi $<$ ni for $1<=\mathrm{i}<=\mathrm{G}$. Thus, the feature is present in top-left portion of $\mathrm{A}$, with $\mathrm{A}_{4}=\mathrm{G}$ and $\mathrm{A}_{2}=$ gi for ith row. Now in $\mathrm{B}$, suppose its signature is given by

$\mathrm{R}_{\mathrm{HxH}} \mathrm{u}_{\mathrm{HxH}} \mathrm{v}_{\mathrm{HxH}} \ldots \ldots \ldots . . \mathrm{R}_{\mathrm{HyH}} \mathrm{u}_{\mathrm{HyH}} \mathrm{v}_{\mathrm{HyH}}$

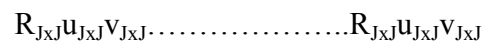

Where, $\mathrm{H}>1, \mathrm{~J}<\mathrm{M}$, (xi $>1$, yi $<$ mi for $\mathrm{H}<=\mathrm{i}<=\mathrm{J})$. Thus the feature is in about the centre of $\mathrm{B}$, with $\mathrm{A}_{4}=(\mathrm{J}-\mathrm{H}+1)$ and $\mathrm{A}_{2}=$ $(y i-x i+1)$ for every ith row.

Now, for any 2 common features, numbers of rows are same. So, $\mathrm{G}=\mathbf{J}-\mathrm{H}+1$. Similarly,

$$
\begin{aligned}
& \sum\left(\left(\mathrm{v}_{\mathrm{ij}}-\mathrm{u}_{\mathrm{ij}}\right)+1,1<=\mathrm{j}<=g \mathrm{gi}\right)=\sum\left(\left(\mathrm{v}_{\mathrm{ij}}-\mathrm{u}_{\mathrm{ij}}\right)+1, \mathrm{xi}<=\mathrm{j}<=\mathrm{yi}\right) . \\
& \left.\left(\mathrm{r}_{\mathrm{ij}}, 1<=\mathrm{j}<=\mathrm{gi}\right)=\mathrm{R}_{\mathrm{ij}}, \mathrm{xi}<=\mathrm{j}<=\mathrm{yi}\right) . \\
& (\mathrm{gi})=(\mathrm{yi}-\mathrm{xi}+1) . \\
& \left(\left(\mathrm{v}_{\mathrm{ij}}-\mathrm{u}_{\mathrm{ij}}\right)+1,1<=\mathrm{j}<=\mathrm{gi}\right)=\left(\left(\mathrm{v}_{\mathrm{ij}}-\mathrm{u}_{\mathrm{ij}}\right)+1, \mathrm{xi}<=\mathrm{j}<=\mathrm{yi}\right) .
\end{aligned}
$$

Again, the relative positions of 2 ranges $r_{1}($ at $(i, j))$ and $r_{2}(a t(k$, 1)) in both of them are same, i.e.
$\left(\mathrm{u}_{\mathrm{ij}}-\mathrm{u}_{\mathrm{kl}}\right)=\left(\mathrm{u}_{\mathrm{IJ}}-\mathrm{u}_{\mathrm{KL}}\right)$ and $\left(\mathrm{v}_{\mathrm{ij}}-\mathrm{v}_{\mathrm{kl}}\right)=\left(\mathrm{v}_{\mathrm{IJ}}-\mathrm{v}_{\mathrm{KL}}\right)$, where $\mathrm{I}=\mathrm{i}+\mathrm{h}$, $\mathrm{J}=\mathrm{j}+\mathrm{k}, \mathrm{K}=\mathrm{k}+\mathrm{h}$ and $\mathrm{L}=\mathrm{l}+\mathrm{k}$. Here, (h, k) show displacement of the feature in different pattern in $\mathrm{x}$ and $\mathrm{y}$ direction.

\section{THE PROPOSED METHOD}

Pattern recognition is done by finding common set of data among set of signatures, each belonging to a particular image. Suppose a pair of images $X_{1}$ and $X_{2}$ have ' $n$ ' common features $x_{1}, x_{2} \ldots . . x_{n}$ and represented by $Y_{1}$ and $Y_{2}$ signatures respectively. Each feature $x_{i}$ has signature $s_{i}$ in first pattern and $S_{i}$ in another one. The motive is to search those signatures. There are 2 methods of searching.

\subsection{Depth First Search}

In DFS, ranges of $s_{i}$ and $S_{i}$ are searched column wise. The process of searching is given below:

1) While raster scanning $Y_{1}$ from the top-left, its each range (along with its $A_{3}$ ) is compared with all the ranges of $Y_{2}$. Let the matched ranges be $r_{1}$ and $r_{2}$ in $Y_{1}$ and $Y_{2}$ respectively.

2) Same procedure is then repeated for the next row in both $\mathrm{Y}_{1}$ and $\mathrm{Y}_{2}$, restricting to those rows only. If the same result is found, the relative positions of matched ranges in $Y_{1}$ and $Y_{2}$ are compared. If they are equal, then from the above observations, combination of those ranges belonging to every signature would be forming a common feature. The portion in $\mathrm{X}_{1}$ and $\mathrm{X}_{2}$ indicated by them is marked and the process from step 2 is again repeated until and unless there is no match found in any particular pair of rows.

3) The above process is repeated for every range in $Y_{1}$ to get compared with all $\mathrm{Y}_{2}$ ranges. When it is all done, we will find that all common features have been marked.

\subsection{Breadth First Search}

It is opposite of DFS. Here, ranges in the signatures of common features are searched row wise. BFS works almost like DFS with a slight change in step 2 where before moving to next rows, ranges next to $r_{1}$ and $r_{2}$ are compared simultaneously until any difference is observed. Rest of the process is same. The result produced by both the methods is same, though time variation can be observed.

As relative position never changes, it is quite obvious for this proposal to find common patterns, each located at any position or one being the part of other pattern. Noisy features can also be handled by putting down the maximum limit over the relative position of 2 ranges in consecutive rows and minimum limit to $\mathrm{A}_{4}$ taken in step 2 to get marked. Thus, the width of common portion would be limited and there would be minimum requirement of height for any feature to get marked in its corresponding pattern.

Its main drawback is that its time consumption is dependent upon the number of ranges. More the variation of colors in any series of pixels more would be the ranges for that row. Also better efficiency would be obtained for patterns having contrast effect with respect to their surroundings so as to avoid any kind of intersection of their boundary ranges with those of latter. 


\section{CONCLUSION}

In this paper, a new technique has been described for pattern recognition. It has been described to find out signatures of various images and compare them to get to their common patterns. Along with BFS and DFS techniques, this algorithm has shown its capability of sorting out feature ordering and noisy features complications. Finally, after discussing its few drawbacks, it has been recommended for continuous tone images with contrast features.

\section{REFERENCES}

[1] Pattern recognition, Guest Editor's introduction, K.S. Fu, Purdue University, May 1976

[2] I.T. Jolliffe "Principle Component Analysis", Springer, New York, 2002

[3] S. Chen and Y. Zhu "Sub-pattern based principle component analysis", Pattern Recognition 37 (2004) 1081-1083

[4] K. Tan and S. Chen "Adaptively weighted sub-pattern PCA for face recognition, Neurocomputing 64 (2005) 505-511

[5] X. Tan, J. Liu and S. Chen "Sub-intrapersonal space analysis for face recognition, Neurocomputing 69 (2006) 1796-1801

"Introductions to Algorithms", II edition, May 2001
[6] Kadappagari Vijaya Kumar and Atul Negi "SubXPCA and a generalization feature partitioning approach to principle component analysis", Pattern Recognition 41 (2008) 1398-1409.

[7] Chengjun Liu and Jian Yang "ICA Color space for pattern recognition", IEEE transactions on neural networks, vol 20, no. 2, February 2009.

[8] P. Comon "Independent component analysis, a new concept?", Signal process, vol. 36, pp 287-314, 1994

[9] J. Karhunen, E. Oja, L. Wang, R. Vigario and J. Joutsensalo "A class of neural networks for independent component analysis", IEEE trans. Neural Netw. Vol 8, no. 3, pp. 486-504, May 1997

[10] Zhang Xiayu, Yu Yongquan, Chen Baixing, Tan Xingxing and Ye Zhiping "Study on extension-based fuzzy pattern recognition", International conference on intelligent and advanced systems 2007

[11] Hong Lian "Adaptive feature learning for information pattern recognition", Proceeding of the international multi-conference on computing in global information theory (ICCGI'2007)

[12] T. Cormen, C. Leiserson, R. Rivest and C. Stien 